\title{
Polycythemia Vera (PV) Complicated by Late Diagnosis of Multiple Splenic Infarcts: A Case Report
}

Musab A Alsubaie ${ }^{1}$, Samer A Alamri ${ }^{1}$, Saleh H Alkhalid ${ }^{1}$

${ }^{1}$ College of Medicine, King Saud bin Abdulaziz University for Health Sciences (KSAU-HS), Jeddah, Saudi

Arabia

\begin{abstract}
Polycythemia vera (PV) is a chronic myeloproliferative tumor where bone marrow makes more red blood cells and other bone marrow cells. Many patients initially are asymptomatic, however, other patients may develop symptoms and complications of this disease later on. One of the PV complications is developing thrombosis in different sites, one of these sites is splenic vessels causing splenic infarction. Here we are describing a JAK2 positive PV case who presented with vague abdominal pain and diagnosed by CT to have splenic infarction, and was treated medically without going to surgical options. In conclusion, splenic infarction is a rare and unique complication of PV. Early diagnosis is paramount to prevent complications and a team approach is highly recommended for the best outcomes.
\end{abstract}

Key words: Polycythemia vera, Splenic infarction, Thrombosis

\section{INTRODUCTION}

Polycythemia vera (PV) is a slow growing myeloproliferative tumor where bone marrow makes more red blood cells and other bone marrow cells, which in turn cause thickening of the blood and thrombosis 1,2. Many patients initially are asymptomatic, however, other patients may develop symptoms of itching, headache, dizziness, minor bleeding or bruising, blurred vision, or shortness of breath ${ }^{1}$. Complications of this disease arise from thickening of the blood and thrombosis, which is the presenting symptom in $20 \%$ of the cases ${ }^{3}$. Sites of thrombosis found to involve both, arteries and veins ${ }^{4}$. One of these sites is the spleen where a single focus of infarction or multiple foci can be developed. Here, we present a case of a patient with PV who had vague abdominal pain for about one month and found lately to have multiple splenic infarcts.

The study was done after approval of ethical board of King Saud bin Abdulaziz university.

\section{Case report}

A 75-year-old female, who is known to have DM and HTN presented to the emergency room (ER) with recurrent epigastric abdominal pain for one month that had increased in severity in the last few days prior to the presentation. The pain was generalized all over the abdomen but increased in central abdomen and was associated with melena for one month. The patient is a known case of PV JAK2 positive on Ruxolitinib $10 \mathrm{mg}$ oral twice daily. She

Received: $02 / 10 / 2017$

Accepted: 12/10/2017

was intolerant to hydroxyurea and was switched to Ruxolitinib 10 months prior to the presentation.

On examination, she was afebrile and vitally stable. The abdomen was soft and lax with severe epigastric tenderness. Her investigations revealed hemoglobin of $7.3 \mathrm{gm} / \mathrm{dl}$ and hematocrit of $24.4 \%$ with low red blood cell count $\left(3.7 \times 10^{12}\right)$, other lab tests were within normal levels. Previous abdominal ultrasound (US) done upon recent ER admission, one month prior to the current visit, showed an enlarged and heterogeneous spleen measuring 13.3 $\mathrm{cm}$ with patent splenic vein and artery.

The patient was given analgesia and pain improved. After that, she had intravenous contrast enhanced CT of the abdomen and pelvis with arterial and venous phases. CT demonstrated an increase in the caliber of splenic and portal veins and its branches with no enhancement representing acute thrombosis. Also, there was superior mesenteric and left ovarian veins thrombosis. In the spleen, there was multiple peripheral wedge shaped subcapsular hypodensities representing multiple splenic infarcts (Figure1).

The patient was admitted as a case of multiple splenic infarctions and was started on heparin infusion. Esophagogastrodudenoscopy (EGD) was done to rule out GI bleeding. It showed the esophagus with large esophageal varices and esophagitis. The patient continued on heparin infusion with therapeutic PTT, and planned to continue for conservative management. Then, she was switched into enoxaparin in addition to warfarin $4 \mathrm{mg}$ once daily and reached a therapeutic range of 
INR. Finally, enoxaparin was stopped and the patient was discharged with full recovery on the same dose of warfarin and planned to follow up as outpatient.

\section{DISCUSSION}

The diagnosis and early detection of PV is very crucial hence prevention of early complications can be taken. PV is diagnosed depending on WHO criteria made in 2008 shown in (table1) ${ }^{5}$. PV complications are divided into four types. Firstly, blood clots complications like: deep vein thrombosis, pulmonary embolism, coronary arteries clots, hepatic blood vessels clots causing Budd Chiary syndrome, and other sites like brain causing strokes and spleen causing its infarction ${ }^{1}$. Secondly, splenomegaly that happens due to viscosity increment, increases the workload on spleen ${ }^{1}$. Thirdly, high red blood cells number which causes opening of gastrointestinal ulcers and bleeding ${ }^{1}$. Fourthly, the overwork of bone marrow can lead to diseases like: myelofibrosis, myelodysplastic syndrome, and acute leukemia ${ }^{1}$. Splenic infarction is rare and can present with left upper quadrant pain or left lower chest pain. Pain can be associated with fever, chills, nausea, vomiting or pleuritic chest pain ${ }^{6}$. Radiological investigations can be very helpful in detecting splenic infarction and US or CT scan can be used. However, US can show false negative results in many cases of splenic infarction that is why CT is more preferred than US when suspecting splenic infarction ${ }^{7}$. There are five possible treatment options in cases of PV. Blood donation is considered a first line therapy in PV and the number of donations depends on how severe is the condition ${ }^{1}$. Secondly, low dose of aspirin 1. Thirdly, hydroxyurea which suppresses bone marrow new red cells production 1. Fourthly, rituximab which destroys the cancerous cells, is used in case if not responding to hydroxyurea ${ }^{1}$. Fifthly, medications that treat itchiness like antihistamine and SSRI are used to achieve the goal of improving quality of life 1. Management of acute splenic infarction is divided into two types, medical and surgical ${ }^{8}$. Medical treatment where supportive therapy was used and close follow up if the patient does not have any complications. Examples of these supportive medications are analgesics like non-steroidal antiinflammatory drugs (NSAID) or narcotics ${ }^{8}$. The benefits of antibiotics and antiplatelet medications (for the treatment of thrombocytosis) have not been formally evaluated ${ }^{8}$. On the other hand, surgical therapy or splenectomy which is used in the presence of any complication, like hematological complications, persistence of symptoms, abscess or sepsis ${ }^{8}$. In cases where splenectomy is difficult due to inflammation around the spleen or adhesions, preoperative splenic artery embolization can be considered or artery ligation ${ }^{8}$. Overall, less data are supporting or preferring certain type of therapy over the other because of the rarity of splenic infarction. In our case, the patient was managed only by medical treatment and was in full recovery upon discharge. In conclusion, splenic infarction is a rare and unique complication of PV. For early diagnosis and treatment, physicians should be aware of such a complication. The majority of patients can be managed conservatively, but the possibility of splenic abscess must be kept in mind, and a team approach to the management is highly recommended.
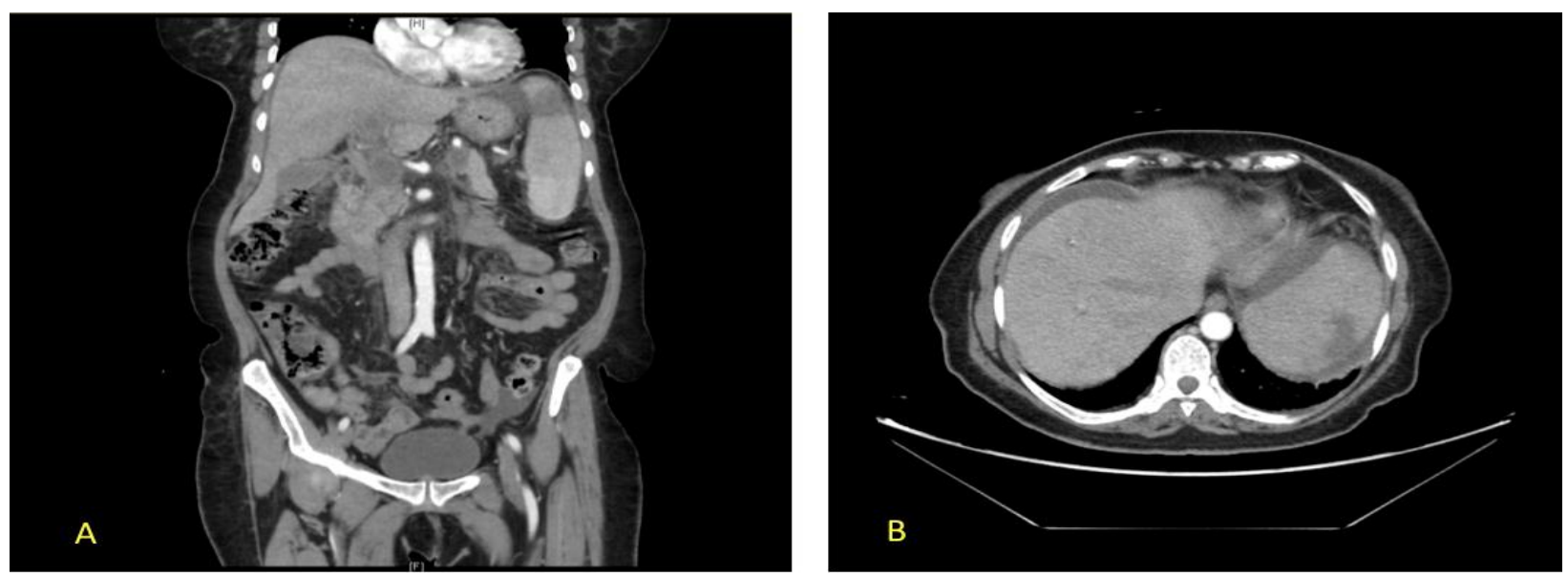

Figure 1: Coronal (A) and axial (B) contrast enhanced CT of the abdomen and pelvis revealing multiple wedgeshaped hypodense areas in the spleen suggesting infarct. 
Table 1: WHO criteria, combination of $\mathrm{A} 1$ and $\mathrm{A} 2$ plus one of the Bs is diagnostic for polycythemia vera. A1 plus 2 Bs is also diagnostic

\begin{tabular}{|l|l|}
\hline A1 & $\begin{array}{l}\text { High red cells mass (more than } 18.5 \mathrm{~g} / \mathrm{dL} \text { in women and more than } 16.5 \mathrm{~g} / \mathrm{dL} \\
\text { in men) }\end{array}$ \\
\hline A2 & Gene mutation (JAK2 V617F or JAK2 exon 12) \\
\hline B1 & Bone marrow tri-lineage myeloproliferation \\
\hline B2 & Subnormal serum erythropoietin levels \\
\hline B3 & Endogenous erythroid colony growth \\
\hline
\end{tabular}

\section{REFERENCES}

1. Mayo Foundation for Medical Education and Research (2017): Polycythemia vera - Symptoms and causes, https://www.mayoclinic.org/diseases-conditions/polycythemia-vera/symptomscauses/syc-20355850

2. Boon Keat Lim (2013): Clinics in diagnostic imaging. Singapore Med J., 54(5): 289-292

3. Gruppo Italiano Studio Policitemia (1995): Polycythemia vera: the natural history of 1213 patients followed for 20 years. Ann Intern Med., 123(9): 656-64

4. Johanna A, Bjorn A, Jan S, Malin H, Elisabeth E, Berit J, Robyn E, Ruben M and Peter J (2013): Patients with polycythemia vera have worst impairment of quality of life among patients with newly diagnosed myeloproliferative neoplasms. Leukemia \& Lymphoma J., 2226-2230

5. Tefferi A (2012): Polycythemia vera and essential thrombocythemia: update on diagnosis, risk stratification, and management. Am J Hematol., 87:285-93

6. Antopolsky M, Hiller N, Salameh S, Goldshtein B and Stalnikowicz R (2009): Splenic infarction: 10 years of experience Am J Emerg Med., 27(3):262-5 .

7. Caremani M, Occhini U, Caremani A, Tacconi D, Lapini L, Accorsi A, Mazzarelli C (2013): Focal splenic lesions: US findings J Ultrasound ,16(2): 65-74

8. Manish $\mathbf{P}$ and Patcher $\mathbf{H}$ (2017): Splenic Infarct Treatment and Management, https://emedicine.medscape.com/article/193718-treatment\#d10 